\title{
Research on fish detection system based on virtual instrument Qirong Lu ${ }^{\mathrm{a}}$, Shiyu Fang ${ }^{\mathrm{b}}$ \\ Guilin University of Technology, Guangzhou 541006, China \\ a330588615@qq.com, b393704066@qq.com
}

Keywords: Fish-finding, ultrasonic sensor, Data acquisition, Auto-Control, LabVIEW.

\begin{abstract}
Through the research on the ultrasonic fish-finding, design a real-time detection system of fish-finding based on LabVIEW and NI PCI-6024E data acquisition card .Under the support of LabVIEW, can be a variety of data real-time acquisition of the fish, through the analysis of the data to provide accurate fish state and displayed in the PC, at the same time the design of an alarm mechanism, necessary to give sound and image warning. The construction of the whole system has realized the function of real-time monitoring and data analysis, which is helpful to improve the efficiency of the fish.
\end{abstract}

\section{Introduction}

In the field of automatic measuring instruments, virtual instrument technology has become the development direction of the testing industry. The idea of "software and instrument", which is put forward by American national instrument company, has been developing for more than 20 years. It is gradually replacing the traditional measurement mode of traditional instrument and meter [1].

This fish detector need a variety of data of underwater fish for real-time monitoring, collection and analysis. The measured data is transmitted to the PC via the network. To achieve these functions, not only the hardware support is required, but also the port resource is rich, and the operation is simple, the data storage and transmission is easy to realize. Considering the factors such as cost ratio, development period and system function expansion, the virtual instrument is a good choice [2].

\section{The principle of fish finder}

Piezoelectric ceramic transducer is used in this system. Piezoelectric ceramic transducers are commonly used to transmit and receive acoustic probes for fish probe. Pressure electric ceramic heat exchanger can the electric energy conversion into sound energy. When ultrasonic wave encounters fish or other small creatures (density is different from water)echo, the echo energy is related to the size of the object density. Echo arrival transducer is converted to voltage signal. After filtering and amplification, according to the signal strength to determine whether the fish.

The echo size of the fish is also known as the fish target strength TS, the unit is $\mathrm{dB}$. The fish target strength is determined by the size of the fish, the type of fish, the working frequency of the sonar and the distance between the fish and the ultrasonic transducer. The reflection model research is mainly on the research of fish maw. Studies have shown that freshwater fish with swimbladders although they account for only $7 \%$ of fish size, but accounted for about $90 \%$ of the reflection intensity. The density of the bones of the fish and the fish is similar to that of water, so it only has a small proportion of total reflection intensity, most of the reflection from the surface of the fish. The target strength but no fish generally have fish swim bladder than the same volume of small 10dB to15dB [3].

The fish body model is from the initial rough cylinder model to the current KRM (Kirchhoff ray mode) model. The model assumes that the fish body is equivalent to a large cylinder filled with gas, which is composed of a water. Using data regression analysis, the fish target strength TS can be expressed as

TS=20 $\log (\mathrm{L})-66(1)$

Where $\mathrm{L}$ said the fish body length, the unit is $\mathrm{cm}$. According to the measured data, the average target strength of the fish body is in a $60 \mathrm{~dB}$ to a $20 \mathrm{~dB}$. Underwater echo intensity RL is a function of underwater terrain and sonar beam angle. For the fish probe can be expressed as 
$\mathrm{RL}=\mathrm{SL}-40 \log (\mathrm{R})+10 \log (\mathrm{A})+10 \log (\mathrm{u})(2)$

SL was the intensity of the ultrasonic wave, and the unit was $\mathrm{dB}$. $\mathrm{R}$ for the transducer surface to the bottom distance. A is the effective beam area, which is determined by the $\mathrm{R}$ and the beam width of the transducer. $10 \log (\mathrm{U})$ is the scattering intensity of the acoustic wave. According to the effect of underwater acoustic reflection.

\section{System hardware design}

The fish detector system is integrated with PC and graphical virtual instrument development environment LabVIEW, which is based on PC machine. The hardware of the system mainly includes: PC LabVIEW, BND-2120 terminal box, PCI-6024E data acquisition card, ultrasonic transducer, transmitter, receiver. System hardware block diagram shown in Figure 1.

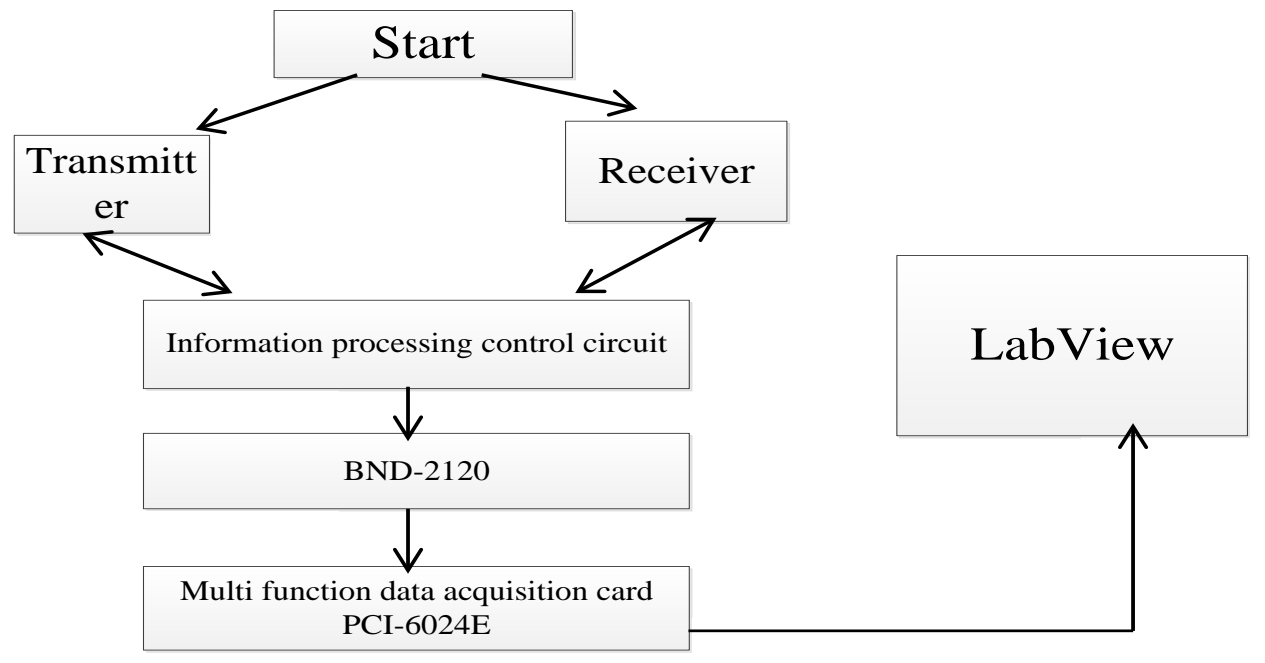

Figure 1 System hardware block diagram

The transmitter generates a pulse frequency of $200 \mathrm{KHz}$,a number of pulses of 25-100,corresponding to a different depth, and a period of 4 times per second pulse. An electronic pulse is emitted by a transmitter, which is converted into sound waves and transmitted into the water. The waves encounter objects (fish, barrier, bottom) then rebound to the transducer, again it will sound is converted into electronic signals are transmitted to the receiver, electronic signal through the information processing circuit processing (including the power amplifier module for signal amplification, after Bandpass filtering module filters to interference signal) finally by acquisition card data acquisition and preservation[4].

\section{System software design}

Fish detector system based on Windows 7 and other operating system, the performance is superior and reliable. The operating mechanism of LabVIEW is a data flow pattern with graphics control flow structure, which can quickly and reliably create multi thread application system [5]. According to the division of the module, the application can be divided into 3 processes, user interface, data acquisition and instrument control. Data acquisition process is shown in Figure 2.

\subsection{Real-time fish monitoring screen}

Real-time fish monitoring module is used to monitor the fish swarm and control equipment. The panel must be able to display a certain amount of information, but also in accordance with the use of field operators. The module is responsible for real-time display of fish and water in the main display area of the user interface. When the user is observing the graphics, the action of the graphic display is transmitted to the module in the form of the message by the interface button control. 


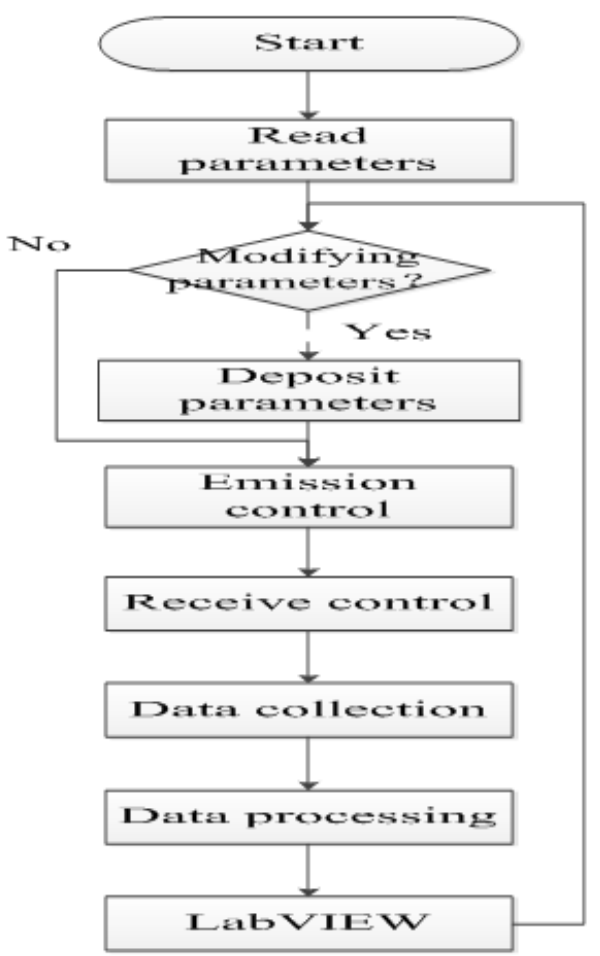

Figure 2 Data acquisition process

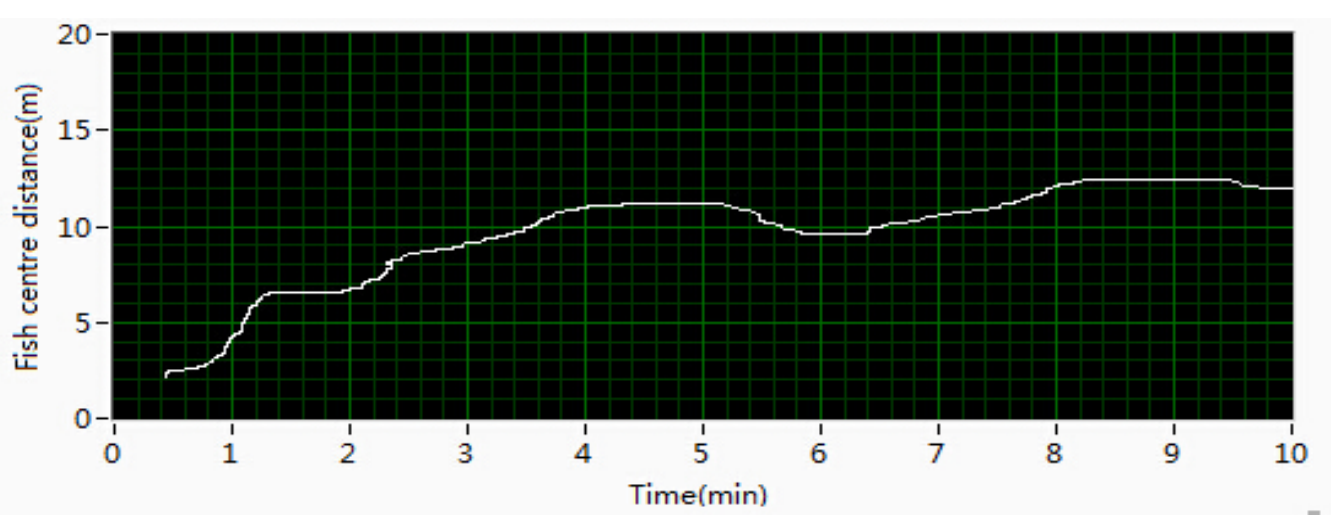

Figure 3 Real-time monitoring of fish swarm distance

\subsection{Data monitoring and analysis}

By selecting different parameters, the error analysis can be carried out and the historical data curve of different characteristics can be obtained, so as to improve the accuracy and sensitivity of the system [6]. This system mainly detects the relationship between the total number of fish and water depth and temperature (Figure 4). The system error, random error and gross error are analyzed and processed.
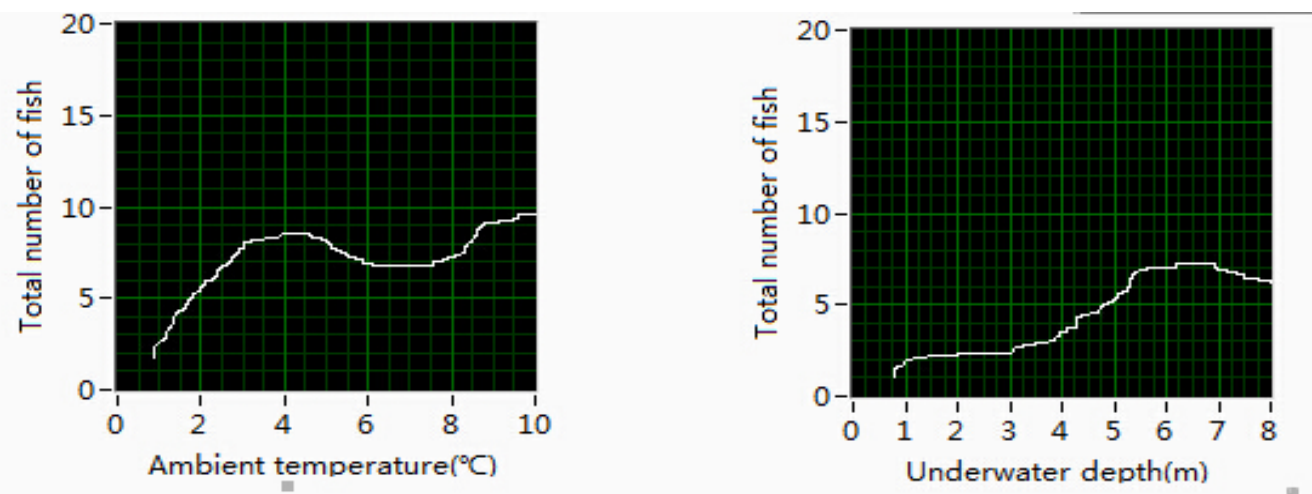

Figure 4 Relationship between fish population and water temperature 


\subsection{Alarm}

The module is responsible for monitoring the situation in the system to monitor the situation changes (including echo signal intensity changes, water temperature changes) issued a warning, in order to remind users. When the echo information is reached, the system sends out the alarm indication and realizes the alarm operation through two ways of sound and light.

\section{Concluding remarks}

The system software is written in LabVIEW, the hardware acquisition part of the NI PCI-6024E data acquisition card. Data acquisition card driver uses NI-DAQ mx LabVIEW driver, through the PCI bus slot and computer connection, data transmission rate is fast, high reliability. LabVIEW provides a powerful, rich interface resources and data processing functions, so that the software interface is more user-friendly, system functions easy to customize and upgrade. With the support of LabVIEW, the user can achieve data acquisition, processing and display of data in real time, and can detect the location and quantity of fish, and provide alarm when necessary.

\section{Reference}

[1]. Hanqiao Huang, Jun Zhou, Xiaozhou Yu. Design and Simulation of missile general test platform based on LabVIEW and DAQ [J].Measurement and control technology, 2009, 28(7):64-67, 71.

[2]. Lujuan Ren, Han Yan. Design of data acquisition system based on LabVIEW and PCI-5124[J].Electronic design engineering, 2010, 18(2): 31-33Lianfang Wang, Xinheng Wu.Design of portable fish detector based on single chip microcomputer [J].Automatic instrument.2008.

[3]. Lianfang Wang, Xinheng Wu.Design of portable fish detector based on single chip microcomputer [J].Automatic instrument.2008.

[4]. Bin Zhang, Hui Dong.Design of fish detector based on microprocessor [J].Computer knowledge and technology.2011.

[5]. YunshuGuo. The design and implementation of the platform for the detection of the fish detector based on LabVIEW [D].Harbin Engineering University.2008.3.

[6]. Lin Sun, Qirong Lu, Yuanyuan Huang.Research on the on-line detection system of gas concentration in process industry [J].Automatic instrument, 2012, 33(3):15-17, 20. 